\title{
Underweight increases the risk of early death in tuberculosis patients
}

\author{
Yung-Feng Yen ${ }^{1,2,3}$, Fu-I Tung ${ }^{3,4}$, Bo-Lung Ho ${ }^{1}$ and Yun-Ju Lai ${ }^{2,5,6 *}$ \\ ${ }^{1}$ Section of Infectious Diseases, Taipei City Hospital, Taipei 103, Taiwan \\ ${ }^{2}$ School of Medicine, National Yang-Ming University, Taipei 112, Taiwan \\ ${ }^{3}$ Center for Infectious Disease and Cancer Research, Kaohsiung Medical University, Kaohsiung 807, Taiwan \\ ${ }^{4}$ Section of Orthopedics, Taipei City Hospital, Taipei City Government, Taipei 103, Taiwan \\ ${ }^{5}$ Division of Endocrinology and Metabolism, Department of Internal Medicine, Puli Branch of Taichung Veterans \\ General Hospital, Nantou 545, Taiwan \\ ${ }^{6}$ Department of Exercise Health Science, National Taiwan University of Sport, Taichung 404, Taiwan
}

(Submitted 17 May 2017 - Final revision received 5 August 2017 - Accepted 27 August 2017-First published online 28 November 2017)

\section{Abstract}

Evidence regarding the association between BMI and mortality in tuberculosis (TB) patients is limited and inconsistent. We investigated the impact of BMI on TB-specific and non-TB-specific mortality with respect to different timing of death. All Taiwanese adults with TB in Taipei were included in a retrospective cohort study in 2012-2014. Multinomial Cox proportional hazards regression was used to evaluate the associations between BMI, cause-specific mortality and timing of death. Of 2410 eligible patients, 86.0\% (2061) were successfully treated, and TB-specific and non-TB-specific mortality occurred for $2 \cdot 2 \%(54)$ and $13.9 \%$ (335), respectively. After controlling for potential confounders, underweight was significantly associated with a higher risk of all-cause mortality (adjusted hazard ratio (AHR) 1.57; 95\% CI 1.26, 1.95), whereas overweight was not. When cause-specific death was considered, underweight was associated with an increased risk of either TB-specific (AHR 1.85; 95\% CI 1.03, 3.33) or non-TB-specific death (AHR 1.52; $95 \%$ CI 1.19, 1.95) during treatment. With joint consideration of cause-specific and timing of death, underweight only significantly increased the risk of TB-specific (AHR 2.23; $95 \%$ CI 1.09, 4.59) and non-TB-specific mortality (AHR 1.81; $95 \%$ CI 1.29, 2.55) within the first 8 weeks of treatment. This study suggests that underweight increases the risk of early death in TB patients during treatment.

\section{Key words: Tuberculosis: BMI: Underweight: Mortality}

Tuberculosis (TB) remains a common and deadly disease globally $^{(1)}$. In 2015, there were an estimated $10 \cdot 4$ million incident cases of TB, and 1.8 million people died from the disease ${ }^{(2)}$.

In Taiwan, of all notified infectious diseases, TB has been the most prevalent for decades ${ }^{(3)}$. Since 2006, Taiwan's Centers for Disease Control (CDC) has adopted a directly observed therapy, short course (DOTS) programme to achieve a successful treatment rate of $85 \%$ and halve TB incidence by 2015 . Since then, the success rate for TB treatment improved only slightly from $64 \cdot 1 \%$ in 2005 to $70.4 \%$ in 2012 . Mortality among TB patients accounted for $81.8 \%$ of the unsuccessful cases of TB treatment ${ }^{(3)}$.

BMI is a popular and useful tool to evaluate the nutritional status of an individual ${ }^{(4)}$. Nutrition disequilibrium (e.g. underweight or obesity) can impair the immune system (e.g. T cell suppression) $)^{(5)}$ and could therefore affect the treatment outcome in TB patients. Although many studies have evaluated the risk factors for mortality in $\mathrm{TB}$ patients ${ }^{(6)}$, the association between BMI and mortality in this population has not been studied extensively, and the existing evidence is inconsistent $^{(4,7-12)}$. Some studies reported that lower BMI was significantly associated with a higher risk of mortality among TB patients ${ }^{(7-10,12)}$, whereas another two found no such an association $^{(4,11)}$. Moreover, one study found that overweight was a protective factor for mortality among TB patients ${ }^{(7)}$.

According to the WHO, mortality in TB patients is defined as death for any reason during treatment ${ }^{(13)}$. However, a number of TB patients die of cerebrovascular disease or malignancy instead of TB. Although many studies have evaluated the factors associated with mortality in TB patients, few studies have determined the predictors of TB-specific or non-TB-specific mortality ${ }^{(6)}$.

According to the CDC guidelines, TB patients should be administered effective drugs for at least 6 months ${ }^{(14)}$. The timing of death in TB patients varies during treatment; for example, many patients die within the first 8 weeks of treatment, whereas others die later ${ }^{(15)}$. Although few studies have examined the prognostic factors for TB with respect to the timing of death ${ }^{(6)}$, a recent report found that predictors of mortality varied according to timing of death in TB patients ${ }^{(16)}$.

Developing effective interventions to improve TB outcomes requires a better understanding of the factors associated

Abbreviations: AHR, adjusted hazard ratio; DOT, directly observed treatment; TB, tuberculosis.

* Corresponding author: Y.-J. Lai, email lailai841081@yahoo.com.tw 
with cause-specific mortality and timing of death. Thus this population-based study aimed to investigate the impact of BMI on TB-specific and non-TB-specific mortality with respect to different timing of death.

\section{Methods \\ Study population and data source}

This retrospective cohort study utilised TB surveillance data from Taipei, Taiwan. In Taipei, TB cases must be reported to the Taipei TB Prevention Center within $7 \mathrm{~d}$ of diagnosis. This study included Taiwanese adults (age $\geq 18$ years) diagnosed with TB in Taipei during the period 2012-2014. TB was defined based on clinical and/or laboratory findings ${ }^{(17)}$. Clinical findings included symptoms (e.g. cough, wasting, prolonged fever) consistent with TB and exclusion of other differential diagnoses based on diagnostic evaluation ${ }^{(17)}$. Laboratory definitions included $M$. tuberculosis isolated from a clinical specimen or acid-fast bacilli (AFB) demonstrated in a clinical specimen from patients with clinical symptoms consistent with TB. This project was approved by the Institutional Review Board of Taipei City Hospitals (TCHIRB- 10505112-E).

\section{Data collection}

When TB patients are reported to the Taipei TB Prevention Center, trained case managers use a structured questionnaire to interview patients about their sociodemographic characteristics, clinical findings, and underlying diseases. Sociodemographic factors include age, sex, BMI, marital status, education level, smoking status, alcohol use and unemployment. TB patients in Taipei are required by law to be monitored until treatment success, death or lost to follow-up. For the purpose of monitoring treatment response, case managers followed up all TB cases by phone or in person once every other week.

\section{Outcome variable}

The outcome variable of interest was treatment outcome, which was categorised as successful treatment or mortality. Mortality was classified as TB-specific or non-TB-specific according to the cause of death. TB-specific death was defined as an underlying cause of death due to TB in the Taiwan Death Certification Registry (International Classification of Diseases (ICD)-9: A010-A018; ICD-10: A15-A19) ${ }^{(18)}$. Non-TB-specific death was defined as any underlying cause of death other than TB. By law, a death certificate must be registered according to ICD 9 or 10 within $30 \mathrm{~d}$ after a patient dies in Taiwan. Because trained medical registrars review all death certificates at the central office of the National Death Certification Registry, the cause-of-death coding in Taiwan is considered very accurate ${ }^{(19)}$.

Mortality was also categorised as early or late according to the timing of death. Early death was defined as death within the first 8 weeks of TB treatment, and late death was defined as mortality later than 8 weeks after the start of TB treatment but before completion of such therapy ${ }^{(20)}$.

\section{Main explanatory variable}

The main explanatory variable was BMI $\left(\mathrm{kg} / \mathrm{m}^{2}\right)$, which was recorded when the case managers interviewed TB cases at the time of TB notification to the Taipei TB Prevention Department. According to the WHO International Classification of adult body weight, BMI was categorised as underweight $\left(<18.5 \mathrm{~kg} / \mathrm{m}^{2}\right)$, normal $\left(18.5-24.9 \mathrm{~kg} / \mathrm{m}^{2}\right)$ or overweight $\left(\geq 25 \mathrm{~kg} / \mathrm{m}^{2}\right)^{(21)}$.

\section{Control variables}

Control variables included sociodemographic factors (education level, smoking status), clinical findings (AFB-smear status, TB culture, cavities on chest radiograph (CXR), pleural effusion, extrapulmonary TB), comorbidities (malignancy, diabetes mellitus, end-stage renal disease) and mode of TB treatment. Education level was categorised as uneducated, elementary school, high school, or university or higher. Smoking status was categorised as never, quit smoking and current smoker. The mode of TB treatment was classified as directly observed treatment (DOT) or self-administered treatment. DOT was defined as administration of antituberculosis medication directly supervised by trained public health observers ${ }^{(22)}$.

\section{Statistical analysis}

First, the sociodemographic characteristics are presented according to BMI category. The two-sample $t$ test was used for comparisons of continuous data between groups. Categorical data were analysed using the Pearson $\chi^{2}$ test, where appropriate.

A Cox proportional hazards regression model was conducted using all-cause mortality $v$. treatment success as the outcome with BMI category as the main explanatory variable. The multinomial Cox proportional hazards regression analysis was used to identify the factors associated with cause-specific mortality (TB-specific $v$. non-TB-specific mortality) and timing of death (early $v$. late death). To investigate the factors associated with cause-specific mortality with respect to timing of death, the treatment outcome in TB patients was classified into five categories: TB-specific death within the first 8 weeks of treatment, TB-specific death later than 8 weeks of treatment, non-TB-specific death within the first 8 weeks of treatment, non-TB-specific death later than 8 weeks of treatment and treatment success. Then multinomial Cox proportional hazards regression analysis was conducted to determine the impact of BMI on TB-specific and non-TB-specific mortality with respect to different timing of death. Adjusted hazard ratios (AHR) and 95\% CI are reported, to indicate the strength and direction of associations. All data management and analyses were performed using the SAS 9.4 software package (SAS Institute).

\section{Results}

\section{Characteristics of patients with tuberculosis}

A total of 2878 TB cases were reported to the Taipei TB Prevention Department in 2012-2014. Of these, ninety-two died before starting TB treatment, twenty-seven were lost to follow-up during treatment, twenty-eight was still on treatment at the time of this study, 212 had incomplete data and 109 had 


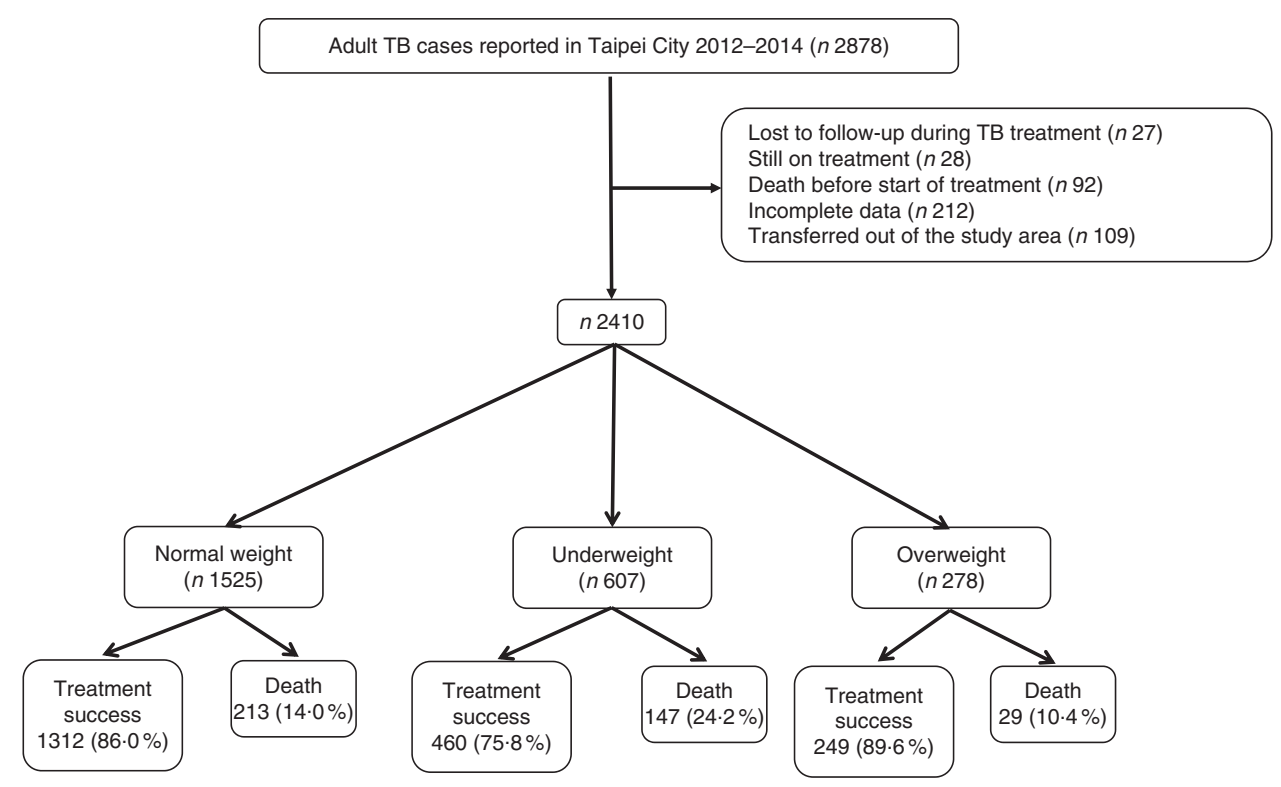

Fig. 1. Study population. TB, tuberculosis.

transferred out of Taipei city (Fig. 1). The remaining 2410 cases were included in the subsequent analysis; the mean age was 64.5 (sD 20.2) years, and $67 \cdot 1 \%$ were men (Table 1 ). According to the WHO BMI definition, $25 \cdot 2,63.3$ and $11.5 \%$ of the patients were classified as underweight, normal weight or overweight, respectively. During the study follow-up period, 145 (14.2\%) deaths occurred in normal weight patients, 99 (24.4\%) deaths occurred in underweight patients, and 19 (10.3\%) deaths occurred in overweight patients (Table 1). Table 1 also shows the distribution of mortality according to cause-specific and timing of death.

\section{Associations between BMI and all-cause mortality}

A Cox proportional hazards model was used to identify independent risk factors for all-cause mortality in TB patients (Table 2). After adjusting for age, sex, clinical findings and comorbidities, the risk of all-cause mortality was significantly higher in underweight patients (AHR 1.57; $95 \%$ CI 1.26, 1.95; $P<0.001)$ than in normal weight patients. Overweight was not significantly associated with all-cause mortality. Other risk factors for all-cause mortality included age $\geq 65$ years (AHR 2.98; $95 \%$ CI 2.10, 4.23), male sex (AHR 1.39; $95 \%$ CI 1.08, 1.78), unemployment (AHR 2.30; $95 \%$ CI 1.43, 3.68), end-stage renal disease (AHR 2.24; $95 \%$ CI 1.54, 3.25), malignancy (AHR 2.68; 95\% CI 2.08, 3.46), AFB-smear positivity (AHR 1.56; $95 \%$ CI $1 \cdot 20,1.95)$, TB-culture positivity (AHR 1.38 ; $95 \%$ CI $1.01,1 \cdot 87$ ) and pleural effusion on CXR (AHR 1.61; 95\% CI 1.23, 2.13). Also, factors associated with a lower risk of all-cause mortality included university or higher education (AHR 0.59; $95 \%$ CI 0.40, $0 \cdot 88$ ) and DOT (AHR 0.62; $95 \%$ CI 0.41, 0.91).

\section{Association between BMI with tuberculosis-specific and non-tuberculosis-specific mortality}

Table 3 shows the results of the multinomial Cox regression analysis of factors associated with TB-specific or non-TB-specific mortality. Underweight was significantly associated with a higher risk of TB-specific (AHR 1.85; 95\% CI 1.03, 3.33;P=0.040) and non-TB-specific mortality (AHR 1.52; $95 \%$ CI 1.19, 1.95; $P<0.001$ ) during treatment. However, overweight was not significantly associated with TB-specific or non-TB-specific death during treatment.

\section{Association between BMI and early or late death during tuberculosis treatment}

Table 4 shows the results of the multinomial Cox regression analysis of factors associated with early or late death during TB treatment. After controlling for potential confounders, underweight was significantly associated with a higher risk of early death (AHR 1.87; 95\% CI 1.38, 2.55; P<0.001), but not late death. Moreover, overweight was not significantly associated with early or late death during TB treatment.

\section{Association between BMI and cause-specific mortality with respect to the timing of death}

Table 5 shows the results of the multinomial Cox regression analysis for the association between BMI and cause-specific mortality in relation to the timing of death. After adjusting for age, sex, clinical findings, and comorbidities, underweight was significantly associated with a higher risk of TB-specific (AHR 2.23; 95\% CI 1.09, 4.59; $P=0.029)$ and non-TB-specific mortality (AHR 1.81; 95\% CI 1.29, 2.55; $P<0.001$ ) within the first 8 weeks of treatment, but not significantly associated with TBspecific or non-TB-specific mortality later than 8 weeks after treatment initiation. Also, overweight was not significantly associated with TB-specific or non-TB-specific death within or later than 8 weeks after treatment initiation.

\section{Discussion}

In this cohort study of 2410 Taiwanese adults with TB in 20122014 , overall mortality was $14.0 \%$. After controlling for potential confounders, underweight was significantly associated with a 
Table 1. Characteristics of tuberculosis patients; by BMI category

(Numbers and percentages; mean values and standard deviations)

\begin{tabular}{|c|c|c|c|c|c|c|c|}
\hline \multirow[b]{3}{*}{ Characteristics } & \multicolumn{6}{|c|}{ BMI $\left(\mathrm{kg} / \mathrm{m}^{2}\right)$} & \multirow[b]{3}{*}{$P$} \\
\hline & \multicolumn{2}{|c|}{ Normal (18.5-24.9) (n 1525) } & \multicolumn{2}{|c|}{ Underweight $(<18.5)(n 607)$} & \multicolumn{2}{|c|}{ Overweight $(\geq 25)(n$ 278) } & \\
\hline & $n$ & $\%$ & $n$ & $\%$ & $n$ & $\%$ & \\
\hline Age (years) & & & & & & & $<0.001$ \\
\hline Mean & \multicolumn{2}{|c|}{$63 \cdot 6$} & \multicolumn{2}{|c|}{$67 \cdot 6$} & \multicolumn{2}{|c|}{63.0} & \\
\hline SD & \multicolumn{2}{|c|}{19.9} & \multicolumn{2}{|c|}{$21 \cdot 3$} & \multicolumn{2}{|c|}{$18 \cdot 2$} & \\
\hline $18-64$ & 722 & $47 \cdot 3$ & 228 & 37.6 & 145 & $52 \cdot 2$ & $<0.001$ \\
\hline$\geq 65$ & 803 & $52 \cdot 7$ & 379 & $62 \cdot 4$ & 133 & $47 \cdot 8$ & \\
\hline Sex & & & & & & & 0.159 \\
\hline Female & 492 & $32 \cdot 3$ & 218 & 35.9 & 84 & $30 \cdot 2$ & \\
\hline Male & 1033 & $67 \cdot 7$ & 389 & $64 \cdot 1$ & 194 & $69 \cdot 8$ & \\
\hline Marital status & & & & & & & 0.02 \\
\hline Unmarried & 293 & 19.2 & 142 & 23.4 & 45 & $16 \cdot 2$ & \\
\hline Married & 1212 & 79.5 & 462 & $76 \cdot 1$ & 227 & $81 \cdot 7$ & \\
\hline Unknown & 20 & 1.3 & 3 & 0.5 & 6 & $2 \cdot 1$ & \\
\hline Education level & & & & & & & 0.192 \\
\hline No education & 115 & 7.5 & 53 & 8.7 & 29 & $10 \cdot 4$ & \\
\hline Elementary school & 325 & $21 \cdot 3$ & 140 & $23 \cdot 1$ & 60 & 21.6 & \\
\hline High school & 577 & 37.8 & 205 & 33.8 & 111 & 39.9 & \\
\hline University or higher & 472 & 31.0 & 187 & $30 \cdot 8$ & 73 & $26 \cdot 3$ & \\
\hline Unknown & 36 & 2.4 & 22 & 3.6 & 5 & 1.8 & \\
\hline Unemployment & & & & & & & $<0.001$ \\
\hline No & 422 & $27 \cdot 7$ & 112 & 18.5 & 81 & $29 \cdot 1$ & \\
\hline Yes & 1103 & $72 \cdot 3$ & 495 & 81.5 & 197 & 70.9 & \\
\hline Smoking status & & & & & & & 0.442 \\
\hline Never smoker & 1208 & $79 \cdot 2$ & 498 & $82 \cdot 0$ & 224 & $80 \cdot 6$ & \\
\hline Former smoker & 99 & 6.5 & 40 & 6.6 & 15 & 5.4 & \\
\hline Current smoker & 218 & $14 \cdot 3$ & 69 & $11 \cdot 4$ & 39 & 14.0 & \\
\hline Any alcohol use & & & & & & & 0.082 \\
\hline No & 1411 & 92.5 & 576 & 94.9 & 254 & 91.4 & \\
\hline Yes & 114 & 7.5 & 31 & $5 \cdot 1$ & 24 & 8.6 & \\
\hline DM & & & & & & & $<0.001$ \\
\hline No & 1265 & 83.0 & 554 & $91 \cdot 3$ & 194 & $69 \cdot 8$ & \\
\hline Yes & 260 & $17 \cdot 0$ & 53 & 8.7 & 84 & 30.2 & \\
\hline ESRD & & & & & & & 0.148 \\
\hline No & 1482 & $97 \cdot 2$ & 586 & 96.5 & 264 & 95.0 & \\
\hline Yes & 43 & $2 \cdot 8$ & 21 & 3.5 & 14 & $5 \cdot 0$ & \\
\hline Malignancy & & & & & & & 0.028 \\
\hline No & 1401 & 91.9 & 552 & 90.9 & 267 & 96.0 & \\
\hline Yes & 124 & $8 \cdot 1$ & 55 & $9 \cdot 1$ & 11 & 4.0 & \\
\hline History of TB & & & & & & & 0.006 \\
\hline No & 1471 & $96 \cdot 5$ & 570 & 93.9 & 272 & $97 \cdot 8$ & \\
\hline Yes & 54 & 3.5 & 37 & $6 \cdot 1$ & 6 & $2 \cdot 2$ & \\
\hline AFB-smear positivity & & & & & & & $<0.001$ \\
\hline No & 958 & $62 \cdot 8$ & 314 & 51.7 & 178 & $64 \cdot 0$ & \\
\hline Yes & 567 & $37 \cdot 2$ & 293 & $48 \cdot 3$ & 100 & $36 \cdot 0$ & \\
\hline TB-culture positivity & & & & & & & $<0.001$ \\
\hline No & 434 & $28 \cdot 5$ & 125 & $20 \cdot 6$ & 85 & $30 \cdot 6$ & \\
\hline Yes & 1091 & 71.5 & 482 & 79.4 & 193 & 69.4 & \\
\hline Cavity on CXR & & & & & & & 0.03 \\
\hline No & 1332 & $87 \cdot 3$ & 511 & 84.2 & 251 & $90 \cdot 3$ & \\
\hline Yes & 193 & $12 \cdot 7$ & 96 & $15 \cdot 8$ & 27 & 9.7 & \\
\hline Pleural effusion & & & & & & & 0.791 \\
\hline No & 1348 & 88.4 & 531 & 87.5 & 247 & 88.9 & \\
\hline Yes & 177 & 11.6 & 76 & 12.5 & 31 & $11 \cdot 1$ & \\
\hline Extrapulmonary TB & & & & & & & $<0.001$ \\
\hline No & 1405 & $92 \cdot 1$ & 577 & $95 \cdot 1$ & 244 & $87 \cdot 8$ & \\
\hline Yes & 120 & $7 \cdot 9$ & 30 & 4.9 & 34 & $12 \cdot 2$ & \\
\hline DOT & & & & & & & 0.08 \\
\hline No & 102 & 6.7 & 46 & $7 \cdot 6$ & 10 & 3.6 & \\
\hline Yes & 1423 & $93 \cdot 3$ & 561 & 92.4 & 268 & $96 \cdot 4$ & \\
\hline Cause-specific mortality & & & & & & & $<0.001$ \\
\hline TB-specific death & 26 & $1 \cdot 7$ & 25 & 4.1 & 3 & $1 \cdot 1$ & \\
\hline Non-TB-specific death & 187 & $12 \cdot 3$ & 122 & $20 \cdot 1$ & 26 & $9 \cdot 3$ & \\
\hline Timing of death & & & & & & & $<0.001$ \\
\hline Early death & 102 & $6 \cdot 7$ & 87 & $14 \cdot 3$ & 17 & $6 \cdot 1$ & \\
\hline Late death & 111 & $7 \cdot 3$ & 60 & 9.9 & 12 & $4 \cdot 3$ & \\
\hline
\end{tabular}

DM, diabetes mellitus; ESRD, end-stage renal disease; TB, tuberculosis; AFB, acid-fast bacilli; CXR, chest radiograph; DOT, directly observed treatment. 
Table 2. Univariate and multivariate analyses of risk factors for all-cause mortality in tuberculosis (TB) patients; Taipei; Taiwan (2012-2014) (Numbers and percentages; hazard ratios (HR), adjusted hazard ratios (AHR) and $95 \%$ confidence intervals)

\begin{tabular}{|c|c|c|c|c|c|c|c|}
\hline \multirow[b]{2}{*}{ Variables } & \multirow[b]{2}{*}{ Number of patients } & \multicolumn{2}{|c|}{ All-cause mortality } & \multicolumn{2}{|c|}{ Univariate analysis } & \multicolumn{2}{|c|}{ Multivariate analysis } \\
\hline & & $n$ & $\%$ & $\mathrm{HR}$ & $95 \% \mathrm{Cl}$ & AHR & $95 \% \mathrm{Cl}$ \\
\hline \multicolumn{8}{|l|}{ BMI $\left(\mathrm{kg} / \mathrm{m}^{2}\right)$} \\
\hline Normal (18.5-24.9) & 1525 & 213 & $14 \cdot 0$ & 1 & & 1 & \\
\hline Underweight $(<18.5)$ & 607 & 147 & $24 \cdot 2$ & $1.86^{\star \star \star}$ & $1.51,2.29$ & $1.57^{\star \star *}$ & $1.26,1.95$ \\
\hline Overweight $(\geq 25)$ & 278 & 29 & $10 \cdot 4$ & 0.74 & $0.50,1.09$ & 0.81 & $0.54,1.19$ \\
\hline \multicolumn{8}{|l|}{ Age (years) } \\
\hline $18-64$ & 1095 & 48 & 4.4 & 1 & & 1 & \\
\hline$\geq 65$ & 1315 & 341 & $25 \cdot 9$ & $6 \cdot 56^{\star * \star}$ & $4.85,8.87$ & $2 \cdot 98^{\star \star \star}$ & $2 \cdot 10,4 \cdot 23$ \\
\hline \multicolumn{8}{|l|}{ Sex } \\
\hline Female & 794 & 95 & $12 \cdot 0$ & 1 & & 1 & \\
\hline Male & 1616 & 294 & $18 \cdot 2$ & $1.53^{\star \star \star}$ & $1.22,1.93$ & $1.39^{\star \star}$ & $1.08,1.78$ \\
\hline \multicolumn{8}{|l|}{ Marital status } \\
\hline Unmarried & 480 & 30 & $6 \cdot 3$ & 1 & & 1 & \\
\hline Married & 1901 & 355 & $18 \cdot 7$ & $3 \cdot 19^{\star \star \star}$ & $2 \cdot 20,4.63$ & 1.32 & $0.89,1.97$ \\
\hline Unknown & 29 & 4 & $13 \cdot 8$ & $2 \cdot 33$ & $0.82,6.62$ & 1.14 & $0.39,3.29$ \\
\hline \multicolumn{8}{|l|}{ Education level } \\
\hline No education & 197 & 46 & 23.4 & 1 & & 1 & \\
\hline Elementary school & 525 & 129 & $24 \cdot 6$ & 1.05 & $0.75,1.47$ & 1.02 & $0.72,1.44$ \\
\hline High school & 893 & 127 & $14 \cdot 2$ & $0.58^{\star \star}$ & $0.41,0.81$ & 0.76 & $0.53,1.08$ \\
\hline University or higher & 732 & 63 & $8 \cdot 6$ & $0.35^{\star \star \star}$ & $0.24,0.52$ & $0.59^{*}$ & $0.40,0.88$ \\
\hline Unknown & 63 & 24 & $38 \cdot 1$ & $1.70^{\star}$ & $1.04,2.78$ & 1.30 & $0.78,2 \cdot 16$ \\
\hline \multicolumn{8}{|l|}{ Unemployment } \\
\hline No & 615 & 21 & 3.4 & 1 & & 1 & \\
\hline Yes & 1795 & 368 & $20 \cdot 5$ & $6.46^{\star \star \star}$ & $4 \cdot 16,10 \cdot 03$ & $2 \cdot 30^{\star \star *}$ & $1.43,3.68$ \\
\hline \multicolumn{8}{|l|}{ Smoking status } \\
\hline Never smoking & 1930 & 329 & $17 \cdot 1$ & 1 & & 1 & \\
\hline Quit smoking & 154 & 28 & $18 \cdot 2$ & 1.05 & $0.71,1.54$ & 0.83 & $0.56,1.23$ \\
\hline Current smoking & 326 & 32 & 9.8 & $0.54^{\star \star \star}$ & $0.37,0.77$ & 0.80 & $0.54,1.19$ \\
\hline \multicolumn{8}{|l|}{ Any alcohol use } \\
\hline No & 2241 & 375 & $16 \cdot 7$ & 1 & & 1 & \\
\hline Yes & 169 & 14 & $8 \cdot 3$ & $0.45^{\star \star}$ & $0.27,0.77$ & 0.72 & $0.41,1.27$ \\
\hline \multicolumn{8}{|l|}{ DM } \\
\hline No & 2013 & 318 & $15 \cdot 8$ & 1 & & 1 & \\
\hline Yes & 397 & 71 & $17 \cdot 9$ & $1 \cdot 11$ & $0.86,1.44$ & 1.09 & $0.84,1.42$ \\
\hline ESRD & & & & & & & \\
\hline No & 2332 & 357 & $15 \cdot 3$ & 1 & & 1 & \\
\hline Yes & 78 & 32 & $41 \cdot 0$ & $3 \cdot 00^{\star \star *}$ & $2.09,4.30$ & $2 \cdot 24^{\star \star *}$ & $1.54,3.25$ \\
\hline Malignancy & & & & & & & \\
\hline No & 2220 & 311 & $14 \cdot 0$ & 1 & & 1 & \\
\hline Yes & 190 & 78 & $41 \cdot 1$ & $3.48^{\star \star \star}$ & $2 \cdot 71,4.46$ & $2 \cdot 68^{\star \star \star}$ & $2.08,3.46$ \\
\hline TB relapse & & & & & & & \\
\hline No & 2313 & 370 & $16 \cdot 0$ & 1 & & 1 & \\
\hline Yes & 97 & 19 & $19 \cdot 6$ & $1 \cdot 17$ & $0.74,1.85$ & $1 \cdot 10$ & $0.69,1.76$ \\
\hline Acid-fast bacilli smear & & & & & & & \\
\hline Negative & 1450 & 189 & $13 \cdot 0$ & 1 & & 1 & \\
\hline Positive & 960 & 200 & $20 \cdot 8$ & $1.65^{\star \star \star}$ & $1 \cdot 35,2 \cdot 02$ & $1.56^{\star \star \star}$ & $1.25,1.94$ \\
\hline TB culture & & & & & & & \\
\hline Negative & 644 & 64 & 9.9 & 1 & & 1 & \\
\hline Positive & 1766 & 325 & 18.4 & $1.95^{\star \star *}$ & $1.49,2.55$ & $1 \cdot 38^{*}$ & $1.01,1.87$ \\
\hline Cavities on CXR & & & & & & & \\
\hline No & 2094 & 349 & $16 \cdot 7$ & 1 & & 1 & \\
\hline Yes & 316 & 40 & $12 \cdot 7$ & 0.73 & $0.52,1.02$ & 0.77 & $0.55,1.08$ \\
\hline Pleural effusion on $\mathrm{CXF}$ & & & & & & & \\
\hline No & 2126 & 321 & $15 \cdot 1$ & 1 & & 1 & \\
\hline Yes & 284 & 68 & $23 \cdot 9$ & $1.68^{* * *}$ & $1 \cdot 29,2 \cdot 18$ & $1 \cdot 61^{* * *}$ & $1 \cdot 23,2 \cdot 13$ \\
\hline Extrapulmonary TB & & & & & & & \\
\hline No & 2226 & 370 & $16 \cdot 6$ & 1 & & 1 & \\
\hline Yes & 184 & 19 & $10 \cdot 3$ & $0.54^{* *}$ & $0.34,0.86$ & 0.87 & $0.54,1.42$ \\
\hline DOT & & & & & & & \\
\hline No & 158 & 28 & $17 \cdot 7$ & 1 & & 1 & \\
\hline Yes & 2252 & 361 & $16 \cdot 0$ & 0.81 & $0.55,1.19$ & $0.62^{*}$ & $0.42,0.91$ \\
\hline
\end{tabular}

$\mathrm{DM}$, diabetes mellitus; ESRD, end-stage renal disease; CXR, chest radiograph; DOT, directly observed treatment. ${ }^{*} P<0.05,{ }^{* *} P<0.01,{ }^{* \star *} P<0.001$ 
Table 3. Associations of BMI with tuberculosis (TB)-specific and non-TB-specific death† (Adjusted hazard ratios (AHR) and $95 \%$ confidence intervals)

\begin{tabular}{|c|c|c|c|c|}
\hline \multirow[b]{2}{*}{ Variables } & \multicolumn{2}{|c|}{ TB-specific death $\ddagger$} & \multicolumn{2}{|c|}{ Non-TB-specific death $\ddagger$} \\
\hline & AHR & $95 \% \mathrm{Cl}$ & AHR & $95 \% \mathrm{Cl}$ \\
\hline \multicolumn{5}{|c|}{ BMI (kg/m²) (normal weight) } \\
\hline Underweight & $1.85^{\star}$ & $1.03,3.33$ & $1.52^{\star \star *}$ & $1.19,1.95$ \\
\hline Overweight & 0.76 & $0.22,2.56$ & 0.81 & $0.54,1.23$ \\
\hline Age $\geq 65$ years & $13 \cdot 86^{\star *}$ & $2 \cdot 40,80 \cdot 02$ & $2 \cdot 55^{\star \star *}$ & $1.72,3.78$ \\
\hline Male sex & 1.14 & $0.59,2.22$ & $1.42^{\star \star}$ & $1.09,1.85$ \\
\hline Married status & $1 \cdot 21$ & $0.33,4.46$ & $1 \cdot 31$ & $0.86,2 \cdot 01$ \\
\hline \multicolumn{5}{|l|}{ Education level } \\
\hline No education & 1 & & 1 & \\
\hline Elementary school & 0.67 & $0.24,1.89$ & 1.07 & $0.73,1.55$ \\
\hline High school & 1.03 & $0.41,2.61$ & 0.71 & $0.49,1.05$ \\
\hline University or higher & 0.99 & $0.35,2 \cdot 81$ & $0.53^{\star *}$ & $0.34,0.83$ \\
\hline Unknown & 0.74 & $0.13,4.18$ & 1.39 & $0.79,2.44$ \\
\hline \multicolumn{5}{|l|}{ Smoking status } \\
\hline Never smoker & 1 & & 1 & \\
\hline Former smoker & 0.92 & $0.33,2.62$ & 0.81 & $0.52,1.26$ \\
\hline Current smoker & 0.54 & $0.12,2.38$ & 0.83 & $0.55,1.24$ \\
\hline Any alcohol use & & & 0.80 & $0.46,1.38$ \\
\hline Unemployment & $2 \cdot 22$ & $0.50,9.92$ & $2 \cdot 29^{\star \star}$ & $1 \cdot 35,3.88$ \\
\hline \multicolumn{5}{|l|}{ HIV infection } \\
\hline DM & 0.64 & $0.26,1.54$ & 1.17 & $0.88,1.55$ \\
\hline ESRD & 1.68 & $0.55,5.13$ & $2 \cdot 32^{\star \star \star}$ & $1.53,3.52$ \\
\hline Malignancy & 1.42 & $0.65,3.15$ & $2 \cdot 94^{\star \star *}$ & $2 \cdot 23,3.89$ \\
\hline History of TB disease & 1.64 & $0.57,4.71$ & 1.02 & $0.60,1.72$ \\
\hline AFB-smear positivity & $2 \cdot 45^{\star \star}$ & $1.31,4.58$ & $1.45^{\star \star}$ & $1.14,1.84$ \\
\hline TB-culture positivity & 1.75 & $0.69,4.42$ & 1.32 & $0.95,1.84$ \\
\hline Cavity on CXR & 1.23 & $0.57,2.65$ & 0.70 & $0.48,1.03$ \\
\hline Pleural effusion & $2 \cdot 39^{*}$ & $1.23,4.66$ & $1.49^{\star}$ & $1.09,2.04$ \\
\hline Extrapulmonary TB & $2 \cdot 17$ & $0.68,6.89$ & 0.75 & $0.42,1.32$ \\
\hline DOT & 0.42 & $0.17,1.01$ & 0.67 & $0.41,1.10$ \\
\hline
\end{tabular}

DM, diabetes mellitus; ESRD, end-stage renal disease; AFB, acid-fast bacilli; CXR, chest radiograph; DOT, directly observed treatment.

${ }^{*} P<0.05,{ }^{* *} P<0.01,{ }^{* * *} P<0.001$.

$\dagger$ Reference is successfully treated individuals.

‡ Adjusted for BMI, age, sex, marital status, education level, unemployment, alcohol use, diabetes mellitus, end-stage renal disease, malignancy, TB relapse, AFB smear, TB culture, cavities on CXR, extrapulmonary TB and directly observed treatment.

higher risk of all-cause mortality, whereas overweight was not. When cause-specific death was considered, underweight was associated with an increased risk of either TB-specific or nonTB-specific mortality during TB treatment. When considering both cause-specific mortality and timing of death, underweight only significantly increased the risk of TB-specific and nonTB-specific mortality within the first 8 weeks of treatment, but was not significantly associated with a higher risk of mortality later than 8 weeks after treatment initiation.

The association of BMI with TB outcome was inconsistent in previous reports ${ }^{(4,7-12)}$. Four studies found that lower BMI was significantly associated with a higher risk of mortality among TB patients ${ }^{(8-10,12)}$, with one study reporting that greater BMI (per unit increase) was significantly associated with a lower risk of mortality ${ }^{(8)}$. In contrast, another two studies found no differences in the risk of mortality based on BMI $\left(<18.5 v . \geq 18.5 \mathrm{~kg} / \mathrm{m}^{2}\right)^{(4,11)}$. Moreover, Hanrahan et l $^{(7)}$ found that underweight significantly increased the risk of mortality among patients with HIV and TB coinfections, whereas obesity and overweight were protective. The present study found that overweight was not significantly associated with the risk of mortality, but underweight was significantly associated with a higher risk of either TB-specific or non-TB-specific mortality during treatment. Furthermore, the main impact of underweight on mortality occurred within the first 8 weeks of TB treatment. The findings in this study indicated that underweight was an independent risk for TB-specific and non-TB-specific mortality, particularly within 8 weeks after the start of TB treatment.

Although underweight was significantly associated with a higher risk of TB-specific and non-TB-specific mortality within the first 8 weeks of TB treatment, the association was NS after the initial 8 weeks of TB treatment. Higher early mortality among underweight TB patients may be due to lower immunity and more severe TB infection in this population. Underweight can suppress lymphocyte stimulation and reduce secretion of Th1 cytokines (e.g. IL-2, TNF- $\alpha$ and interferon- $\gamma)^{(23)}$, which could cause more severe TB infection. In malnourished animals, macrophages produce more transforming growth factor $\beta$, which further suppresses $\mathrm{T}$ cells and causes severe TB disease ${ }^{(24,25)}$. Animal studies have also shown that malnourished mice have an impaired immune system (e.g. reduction of reactive $\mathrm{N}$ intermediates) in response to Mycobacterium infection ${ }^{(26)}$. Moreover, malnourished animals have higher bacterial burdens with TB infection and die earlier of infection ${ }^{(26)}$.

This study showed that overweight TB patients had a lower risk of all-cause mortality than normal weight patients $(10.4 v$. $14.0 \%$ ), although this was not statistically significant. Although this association of interest has not been extensively studied, 
Table 4. Associations of BMI with early and late death in tuberculosis (TB) patients $\dagger$ (Adjusted hazard ratios (AHR) and $95 \%$ confidence intervals)

\begin{tabular}{|c|c|c|c|c|}
\hline \multirow[b]{2}{*}{ Variables } & \multicolumn{2}{|c|}{ Early death $\ddagger$} & \multicolumn{2}{|c|}{ Late death $\ddagger$} \\
\hline & AHR & $95 \% \mathrm{Cl}$ & AHR & $95 \% \mathrm{Cl}$ \\
\hline \multicolumn{5}{|c|}{ BMI (kg/m²) (normal weight) } \\
\hline Underweight & $1.87^{\star \star *}$ & $1.38,2.55$ & 1.26 & $0.90,1.76$ \\
\hline Overweight & 1.04 & $0.61,1.76$ & 0.60 & $0.34,1.09$ \\
\hline Age $\geq 65$ years & $2 \cdot 60^{\star \star *}$ & $1.56,4.32$ & $3 \cdot 54^{\star \star \star}$ & $1.95,6 \cdot 41$ \\
\hline Male sex & 1.33 & $0.95,1.85$ & $1.47^{\star}$ & $1.02,2.11$ \\
\hline Married status & $2 \cdot 28^{\star}$ & $1 \cdot 16,4.45$ & 0.82 & $0.48,1.41$ \\
\hline \multicolumn{5}{|l|}{ Education level } \\
\hline No education & 1 & & 1 & \\
\hline Elementary school & 0.84 & $0.53,1.33$ & 1.31 & $0 \cdot 76,2 \cdot 26$ \\
\hline High school & 0.64 & $0.40,1.01$ & 0.93 & $0.53,1.62$ \\
\hline University or higher & $0.50^{\star}$ & $0.29,0.85$ & 0.76 & $0.40,1.43$ \\
\hline Unknown & 0.84 & $0.40,1.73$ & 2.02 & $0.94,4.31$ \\
\hline \multicolumn{5}{|l|}{ Smoking status } \\
\hline Never smoker & 1 & & 1 & \\
\hline Former smoker & 0.80 & $0.44,1.45$ & 0.81 & $0.44,1.47$ \\
\hline Current smoker & 0.84 & $0.49,1.44$ & 0.73 & $0.42,1.29$ \\
\hline Any alcohol use & 0.62 & $0.27,1.43$ & 0.87 & $0.42,1.79$ \\
\hline Unemployment & $2 \cdot 60^{\star \star}$ & $1 \cdot 27,5 \cdot 31$ & $2 \cdot 00^{\star}$ & $1.00,4.01$ \\
\hline DM & 0.97 & $0.66,1.43$ & 1.25 & $0.86,1.83$ \\
\hline ESRD & 1.51 & $0.79,2.87$ & $3 \cdot 15^{\star \star \star}$ & $1 \cdot 85,5 \cdot 36$ \\
\hline Malignancy & $2 \cdot 30^{\star \star *}$ & $1 \cdot 60,3.31$ & $3 \cdot 46^{\star \star \star}$ & $2 \cdot 34,5 \cdot 12$ \\
\hline History of TB disease & 1.00 & $0.50,1.96$ & 1.28 & $0.65,2.52$ \\
\hline AFB-smear positivity & $2 \cdot 23^{\star \star \star}$ & $1.63,3.06$ & 1.05 & $0.74,1.49$ \\
\hline TB-culture positivity & $1 \cdot 79^{\star}$ & $1.10,2.91$ & $1 \cdot 11$ & $0.72,1.73$ \\
\hline Cavity on CXR & 0.94 & $0.61,1.44$ & $0.56^{\star}$ & $0.32,0.99$ \\
\hline Pleural effusion & $2 \cdot 00^{\star \star *}$ & $1.37,2.91$ & 1.29 & $0.83,1.99$ \\
\hline Extrapulmonary TB & 1.38 & $0.72,2.66$ & 0.55 & $0.26,1.15$ \\
\hline DOT & $0.32^{\star \star *}$ & $0.20,0.51$ & 3.20 & $0.97,10.62$ \\
\hline
\end{tabular}

DM, diabetes mellitus; ESRD, end-stage renal disease; AFB, acid-fast bacilli; CXR, chest radiograph; DOT, directly observed treatment.

* $P<0.05 ; * * 20.01 ; * \star * * 0.001$.

$\dagger$ Reference is successfully treated individuals.

$\ddagger$ Adjusted for BMI, age, sex, marital status, education level, unemployment, alcohol use, diabetes mellitus, end-stage renal disease, malignancy,

TB relapse, AFB smear, TB culture, cavities on CXR, extrapulmonary TB and directly observed treatment.

Table 5. Multinomial regression for the association between BMI and cause-specific mortality in relation to timing of death in tuberculosis (TB) patients $\dagger$ (Adjusted hazard ratios (AHR) and $95 \%$ confidence intervals)

\begin{tabular}{|c|c|c|c|c|c|c|c|c|}
\hline \multirow[b]{3}{*}{ Variables } & \multicolumn{4}{|c|}{ TB-specific mortality $\ddagger$} & \multicolumn{4}{|c|}{ Non-TB-specific mortality $\ddagger$} \\
\hline & \multicolumn{2}{|c|}{ Early death } & \multicolumn{2}{|c|}{ Late death } & \multicolumn{2}{|c|}{ Early death } & \multicolumn{2}{|c|}{ Late death } \\
\hline & AHR & $95 \% \mathrm{Cl}$ & AHR & $95 \% \mathrm{Cl}$ & AHR & $95 \% \mathrm{Cl}$ & AHR & $95 \% \mathrm{Cl}$ \\
\hline \multicolumn{9}{|c|}{ BMI $\left(\mathrm{kg} / \mathrm{m}^{2}\right)$ (normal weight) } \\
\hline Underweight & $2 \cdot 23^{*}$ & $1.09,4.59$ & 1.24 & $0.41,3.74$ & $1.81^{\star \star *}$ & $1 \cdot 29,2 \cdot 55$ & 1.25 & $0.88,1.79$ \\
\hline Overweight & 1.21 & $0.33,4.35$ & - & & 1.01 & $0.56,1.81$ & 0.66 & $0.36,1.19$ \\
\hline
\end{tabular}

${ }^{*} P<0.05,{ }^{* \star *} P<0.001$.

$\dagger$ Reference is successfully treated individuals.

‡ Variables included: age, sex, clinical findings and comorbidities.

a prior study found that overweight or obesity was protective against mortality in patients with HIV-TB co-infection ${ }^{(7)}$. Patients with TB who experience a mild or moderate increase in BMI may consume more protein and energy on a daily basis, which could result in more robust immune function and reduce mortality. However, more studies are needed to evaluate the impact of overweight or obesity on all-cause and cause-specific mortality as well as timing of death in TB patients.

This study found that DOT was significantly associated with a lower risk of mortality after controlling for BMI and other potential confounders. In Taipei's DOTS programme, each DOT observer monitors five to fifteen TB patients on average ${ }^{(3)}$. DOT observers interview patients regarding their TB symptoms and complications of treatment under the supervision of public health nurses. When TB patients on DOT have, for example, worsened dyspnea, the public health nurses contact doctors to arrange hospital visits. DOT has been recommended for TB patients to improve their adherence to treatment ${ }^{(27)}$. Our study suggests that DOT programme should be applied to all TB patients to further reduce mortality. 
The strength of this study is that all eligible TB patients were included in the analysis; therefore, the sample size was not based on the considerations of statistical power. However, some limitations should be considered when interpreting the findings of this citywide population-based study. First, in this retrospective cohort study, some important patient information (e.g. intravenous drug use, chronic lung disease and psychiatric disorders) was not available. Second, $25.4 \%$ of the TB cases were identified based on clinical diagnosis rather than by AFB smear or culture, which could have resulted in over-diagnosis of TB. However, this is less likely in this study because the Taipei TB Control Department holds an expert committee monthly to discuss the uncertain diagnosis of $\mathrm{TB}$ cases $^{(3)}$. Third, the information regarding BMI among TB patients were self-reported, and therefore subject to recall bias. Moreover, this study only measured BMI at baseline. As BMI can change during TB treatment ${ }^{(28)}$, more studies are needed to determine the time-varying effect of BMI on mortality in TB patients. Finally, the external validity of our findings may be a concern because all the participants were Taiwanese. The generalisability of our results to other non-Asian ethnic groups thus requires further verification. Nevertheless, our findings suggest new avenues for future research.

\section{Conclusions}

In the present study, mortality was high in TB patients in Taipei, Taiwan, in 2012-2014. After controlling for other covariates, underweight was significantly associated with an increased risk of all-cause mortality during TB treatment, whereas overweight was not. For cause-specific mortality, underweight was associated with an increased risk of either TB-specific or non-TB-specific mortality during treatment. With the simultaneous consideration of cause-specific mortality and timing of death, underweight only significantly increased the risk of TB-specific and non-TB-specific mortality within the first 8 weeks of treatment, but was not significantly associated with a higher risk of mortality after the initial 8 weeks of treatment. The findings in this study indicate that underweight increases the risk of early death in TB patients during the treatment.

\section{Acknowledgements}

The authors are grateful to registered nurses Jen-Chieh Hsiao and Miao-Yun Chen for interviewing the subjects and reviewing their medical records.

This study was funded.

Y.-F. Y., F.-I. T., B.-L. H., Y.-J. L. substantially contributed to the conception and design of the study, data analysis, data interpretation and the drafting of the manuscript. Y.-F. Y. substantially contributed to data acquisition and interpretation of the results. Y.-F. Y., F.-I. T., B.-L. H., Y.-J. L. all approved the final version of the manuscript.

The authors declare that there are no conflicts of interest.

\section{References}

1. Lyons JG \& Stewart S (2013) Commentary: tuberculosis, diabetes and smoking: a burden greater than the sum of its parts. Int J Epidemiol 42, 230-232.
2. World Health Organization (2017) Global tuberculosis control. WHO report 2017. http://www.searo.who.int/tb/documents/ annual_tb_repot_2017/en/ (accessed August 2017).

3. Taiwan Centers for Disease Control and Prevention (2017) [Promulgated Definitions of TB]. Taipei: CDC; http://nidss. cdc.gov.tw/singledisease $\cdot$ asp $\mathrm{x} ? \mathrm{pt}=\mathrm{s} \& \mathrm{dc}=1 \& \mathrm{dt}=3 \&$ disease $=010$ (accessed February 2017) (in Chinese).

4. Kim DK, Kim HJ, Kwon SY, et al. (2008) Nutritional deficit as a negative prognostic factor in patients with miliary tuberculosis. Eur Respir J 32, 1031-1036.

5. Schaible UE \& Kaufmann SH (2007) Malnutrition and infection: complex mechanisms and global impacts. PLoS Med 4, e115.

6. Waitt CJ \& Squire SB (2011) A systematic review of risk factors for death in adults during and after tuberculosis treatment. Int J Tuberc Lung Dis 15, 871-885.

7. Hanrahan CF, Golub JE, Mohapi L, et al. (2010) Body mass index and risk of tuberculosis and death. AIDS 24, 1501-1508.

8. Bhargava A, Chatterjee M, Jain Y, et al. (2013) Nutritional status of adult patients with pulmonary tuberculosis in rural central India and its association with mortality. PLOS ONE $\mathbf{8}$, e77979.

9. Zachariah R, Spielmann MP, Harries AD, et al. (2002) Moderate to severe malnutrition in patients with tuberculosis is a risk factor associated with early death. Trans $R$ Soc Trop Med Hyg 96, 291-294.

10. Yen YF, Chuang PH, Yen MY, et al. (2016) Association of body mass index with tuberculosis mortality: a populationbased follow-Up study. Medicine (Baltimore) 95, e2300.

11. Kwon YS, Kim YH, Song JU, et al. (2014) Risk factors for death during pulmonary tuberculosis treatment in Korea: a multicenter retrospective cohort study. J Korean Med Sci $\mathbf{2 9}$, $1226-1231$.

12. Lai HH, Lai YJ \& Yen YF (2017) Association of body mass index with timing of death during tuberculosis treatment. PLOS ONE 12, e0170104.

13. Balabanova Y, Drobniewski F, Fedorin I, et al. (2006) The Directly Observed Therapy Short-Course (DOTS) strategy in Samara Oblast, Russian Federation. Respir Res 7, 44.

14. American Thoracic Society (2003) Treatment of tuberculosis. MMWR Recomm Rep 52, 1-77.

15. Jonnalagada S, Harries AD, Zachariah R, et al. (2011) The timing of death in patients with tuberculosis who die during anti-tuberculosis treatment in Andhra Pradesh, South India. BMC Public Health 11, 921.

16. Yen YF, Yen MY, Shih HC, et al. (2013) Prognostic factors associated with mortality before and during anti-tuberculosis treatment. Int J Tuberc Lung Dis 17, 1310-1316.

17. Wang FD, Chang $\mathrm{CH}$, Su WJ, et al. (2006) Screening of hospital workers for pulmonary tuberculosis in a medical center in Taiwan. Infect Control Hosp Epidemiol 27, 510-511.

18. Department of Health (1971) Vital Statistics in Taiwan. Taiwan: Department of Health, Executive Yuan.

19. Lu TH, Lee MC \& Chou MC (2000) Accuracy of cause-of-death coding in Taiwan: types of miscoding and effects on mortality statistics. Int J Epidemiol 29, 336-343.

20. Nguyen LT, Hamilton CD, Xia Q, et al. (2011) Mortality before or during treatment among tuberculosis patients in North Carolina, 1993-2003. Int J Tuberc Lung Dis 15, 257-262.

21. World Health Organization (1995) Physical Status: The Use and Interpretation of Anthropometry. Report of a WHO Expert Committee. Geneva: WHO. http://whqlibdoc.who.int/trs/ WHO_TRS_854.pdf

22. Jasmer RM, Seaman CB, Gonzalez LC, et al. (2004) Tuberculosis treatment outcomes: directly observed therapy compared with self-administered therapy. Am J Respir Crit Care Med 170, 561-566 
23. Cegielski JP \& McMurray DN (2004) The relationship between malnutrition and tuberculosis: evidence from studies in humans and experimental animals. Int J Tuberc Lung Dis $\mathbf{8}$, 286-298.

24. Dai G \& McMurray DN (1999) Effects of modulating TGFbeta 1 on immune responses to mycobacterial infection in guinea pigs. Tuber Lung Dis 79, 207-214.

25. Dai G \& McMurray DN (1998) Altered cytokine production and impaired antimycobacterial immunity in proteinmalnourished guinea pigs. Infect Immun 66, 3562-3568.
26. Chan J, Tian Y, Tanaka KE, et al. (1996) Effects of protein calorie malnutrition on tuberculosis in mice. Proc Natl Acad Sci U S A 93, 14857-14861.

27. Frieden TR \& Sbarbaro JA (2007) Promoting adherence to treatment for tuberculosis: the importance of direct observation. Bull World Health Organ 85, 407-409.

28. Vasantha M, Gopi PG \& Subramani R (2009) Weight gain in patients with tuberculosis treated under directly observed treatment short-course (DOTS). Indian J Tuberc $\mathbf{5 6}$, 5-9. 